OPEN ACCESS

Edited by:

Peng Luo,

South China Sea Institute

of Oceanology (CAS), China

Reviewed by:

Gloria Paz Levicán,

Universidad de Santiago de Chile,

Chile

Michael P. Ryan,

University of Limerick, Ireland

*Correspondence:

William J. Hickey

wjhickey@wisc.edu

Specialty section:

This article was submitted to

Evolutionary and Genomic

Microbiology,

a section of the journal

Frontiers in Microbiology

Received: 24 April 2018

Accepted: 20 June 2018

Published: 11 July 2018

Citation:

Obi CC, Vayla S, de Gannes V,

Berres ME, Walker J, Pavelec D,

Hyman J and Hickey WJ (2018) The

Integrative Conjugative Element clc

(ICEClC) of Pseudomonas aeruginosa

JB2. Front. Microbiol. 9:1532.

doi: 10.3389/fmicb.2018.01532

\section{The Integrative Conjugative Element clc (ICEclc) of Pseudomonas aeruginosa JB2}

\author{
Chioma C. Obi ${ }^{1}$, Shivangi Vayla ${ }^{2}$, Vidya de Gannes ${ }^{3}$, Mark E. Berres $^{4}$, Jason Walker ${ }^{4}$, \\ Derek Pavelec ${ }^{4}$, Joshua Hyman ${ }^{4}$ and William J. Hickey ${ }^{2 *}$
}

${ }^{1}$ Department of Biological Sciences, Bells University of Technology, Ota, Nigeria, ${ }^{2}$ Department of Soil Science, University of Wisconsin-Madison, Madison, WI, United States, ${ }^{3}$ Department of Food Production, University of the West Indies,

St. Augustine, Trinidad and Tobago, ${ }^{4}$ Biotechnology Center, University of Wisconsin-Madison, Madison, WI, United States

Integrative conjugative elements (ICE) are a diverse group of chromosomally integrated, self-transmissible mobile genetic elements (MGE) that are active in shaping the functions of bacteria and bacterial communities. Each type of ICE carries a characteristic set of core genes encoding functions essential for maintenance and self-transmission, and cargo genes that endow on hosts phenotypes beneficial for niche adaptation. An important area to which ICE can contribute beneficial functions is the biodegradation of xenobiotic compounds. In the biodegradation realm, the best-characterized ICE is ICEclc, which carries cargo genes encoding for ortho-cleavage of chlorocatechols (clc genes) and aminophenol metabolism (amn genes). The element was originally identified in the 3-chlorobenzoate-degrader Pseudomonas knackmussii B13, and the closest relative is a nearly identical element in Burkholderia xenovorans LB400 (designated ICEclc-B13 and ICEclc-LB400, respectively). In the present report, genome sequencing of the o-chlorobenzoate degrader Pseudomonas aeruginosa JB2 was used to identify a new member of the ICEclc family, ICEclc-JB2. The cargo of ICEclc-JB2 differs from that of ICEclc-B13 and ICEclc-LB400 in consisting of a unique combination of genes that encode for the utilization of $o$-halobenzoates and o-hydroxybenzoate as growth substrates (ohb genes and hyb genes, respectively) and which are duplicated in a tandem repeat. Also, ICEclc-JB2 lacks an operon of regulatory genes (tciR-marR-mfsR) that is present in the other two ICEclc, and which controls excision from the host. Thus, the mechanisms regulating intracellular behavior of ICEclc-JB2 may differ from that of its close relatives. The entire tandem repeat in ICEclc-JB2 can excise independently from the element in a process apparently involving transposases/insertion sequence associated with the repeats. Excision of the repeats removes important niche adaptation genes from ICEclc-JB2, rendering it less beneficial to the host. However, the reduced version of ICEclc-JB2 could now acquire new genes that might be beneficial to a future host and, consequently, to the survival of ICEclc-JB2. Collectively, the present identification and characterization of ICEclc-JB2 provides insights into roles of MGE in bacterial niche adaptation and the evolution of catabolic pathways for biodegradation of xenobiotic compounds.

Keywords: integrative conjugative element (ICE), ICEclc, biodegradation, xenobiotic metabolism, PCBs, chlorobenzoates, Pseudomonas aeruginosa 


\section{INTRODUCTION}

Integrative conjugative elements (ICE) are a diverse group of chromosomally integrated mobile genetic elements (MGE) that are active in shaping the behavior of bacteria and bacterial communities (Wozniak and Waldor, 2010). ICE are selftransmissible from host chromosomes, and each type of ICE carries a characteristic set of core genes that encode for its excision, circularization, conjugative transfer and site-specific integration in a new host (Johnson and Grossman, 2015; Banuelos-Vazquez et al., 2017; Cury et al., 2017; Delavat et al., 2017). The other components of ICE are the cargo genes, which encode functions affecting bacterial life styles and niche adaptation. ICEs have been most extensively studied with respect to their roles in conferring virulence factors and/or resistance to antimicrobial compounds (Chowdhury et al., 2016; Clawson et al., 2016; Leon-Sampedro et al., 2016; Olaitan et al., 2016; Bie et al., 2017; Chuzeville et al., 2017; Ryan et al., 2017; Sugimoto et al., 2017; Vanneste, 2017; Zhou et al., 2017). But, the spectrum of ICE-encoded adaptation functions is broad and also includes resistance to heavy metals (Colombi et al., 2017; Harmer et al., 2017), rhizobial nodulation functions (Ling et al., 2016), biofilm formation characteristics (Wang et al., 2017) and components of metabolic pathways (Gaillard et al., 2006; Zamarro et al., 2016; Suenaga et al., 2017).

Biodegradation of xenobiotic compounds is a key environmental service of bacterial communities, and MGE are well-established as playing a central role in the evolution of metabolic capacity essential for these activities (Top et al., 2002; Top and Springael, 2003; Diaz, 2004; Shintani et al., 2010). While initial work exploring MGE centered largely on plasmids, the advent of genome sequencing has revealed the role of ICE and other types of genomic islands (van der Meer and Sentchilo, 2003; Gaillard et al., 2006; Hickey et al., 2012; Chong et al., 2014; Pathak et al., 2016; Zamarro et al., 2016; Suenaga et al., 2017).

In the biodegradation realm, the best-characterized ICE is termed ICEclc and was originally identified in the 3chlorobenzoate-degrader Pseudomonas knackmussii B13 (Gaillard et al., 2006). Integration of ICEclc is mediated by an integrase that is located a terminus of the element (IntB13). Within ICEclc, genes encoding core functions (maintenance and stability of the element) are segregated to one side while those encoding biodegradation functions are grouped to the other (Gaillard et al., 2006). Biodegradation functions encoded by ICEclc include the pathway for orthocleavage of chlorocatechols (clc genes) and amp genes encoding aminophenol metabolism (Gaillard et al., 2006). A number of other ICE and genomic islands are related to ICEclc by synteny in core functions (Gaillard et al., 2006), and one of these that occurs in Burkholderia xenovorans LB400 (currently Paraburkholderia xenovorans LB400, (Sawana et al., 2014)) is identical except for an additional ca. $1 \mathrm{~kb}$ of cargo and one extra gene in core region; this element is hereafter referred to as ICEclc-LB400 to distinguish it from the original ICEclc (hereafter referred to as ICEclc-B13).

Polychlorinated biphenyls (PCBs) are an important group of environmental contaminants for which biodegradation is a primary pathway of removal (Field and Sierra-Alvarez, 2008).
However, bacteria that effect these transformations typically do so by cometabolism (i.e., growth on biphenyl), in part because they cannot utilize as growth substrates the chlorinated benzoic acids produced from PCB breakdown (Field and SierraAlvarez, 2008). Thus, organisms such as the chlorobenzoatedegrader Pseudomonas aeruginosa JB2 (Hickey and Focht, 1990) can enhance PCB mineralization when growing alongside the PCB-cometabolizers (Hickey et al., 1993). It's also possible to create more effective PCB-degraders by introducing into these organisms genes that encode chlorobenzoate metabolism. Generation of such hybrids could be greatly facilitated if genes of interest are naturally associated with MGE.

The $o h b$ genes encoding degradation of a range of orthochlorobenzoates have been identified in $P$. aeruginosa JB2 (Hickey and Sabat, 2001) and demonstrated to transfer from P. aeruginosa JB2 to other bacteria (Perezlesher and Hickey, 1995; Hickey et al., 2001). Moreover, bacteria acquiring the ohb genes concomitantly acquired $c l c$ genes and $h y b$ genes encoding for the metabolism of ortho-hydroxybenzoate (Hickey et al., 2001). The goal of this study was to identify MGE that are associated with these genes in the genome of $P$. aeruginosa JB2.

\section{MATERIALS AND METHODS}

\section{DNA Preparation}

Pseudomonas aeruginosa JB2 was grown on 2-chlorobenzoate (2-CBa) as described previously (Hickey and Focht, 1990) and cells harvested in late log phase for use in genomic DNA preparations. Two approaches were used for genomic DNA (gDNA) preparation from $P$. aeruginosa JB2 that were compatible with the sequencing technology applied. For use in short read sequencing (Illumina $250 \mathrm{HiSeq}$ ) and long-read sequencing by single molecule, real-time sequencing (SMRT) using PacBio technology, gDNA was prepared by using an Illustra Bacterial DNA Minispin kit (GE Healthcare) following the manufacturer's suggested protocol. For nanopore DNA strand sequencing (Oxford Nanopore Technologies, ONT), DNA was extracted from approximately $5 \times 10^{6}$ cells of $P$. aeruginosa by using the MagAttract HMW DNA Kit (Qiagen) following the manufacturer's instructions. The quality of all gDNA preparations was assessed with a Qubit fluorometer (Invitrogen) to quantify nucleic acid concentration, and with a Fragment Analyzer (Advanced Analytical Technologies, Inc.) using the High Sensitivity Large Fragment $50 \mathrm{~Kb}$ Analysis Kit to determine the fragment size distribution.

\section{Library Preparation and Sequencing}

The PacBio sequencing library was prepared following the AccelNGS XL Library Kit for PacBio protocol (Swift Biosciences). The resulting library was size-selected to $20 \mathrm{~kb}$ with a PippenHT (Sage Science). A final library QC was performed with a Qubit fluorometer to quantify library concentration and fragment size distribution of the library was determined on a Fragment Analyzer (Advanced Analytical Technologies, Inc.) using the High Sensitivity Large Fragment 50 kb Analysis Kit. The prepared sequence library was loaded onto a single Sequel v 2.1 SMRTcell 
at a concentration of $6 \mathrm{pM}$, following the PacBio diffusion loading protocol and including a polymerase-bound complex cleanup. One 600-min movie was taken of the SMRTcell. Illumina sequencing was done with an Illumina regular fragment library.

For ONT sequencing, ca. 1,500 ng of gDNA was used as input for the 1D genomic DNA by ligation (SQK-LSK108) protocol version GDE_9002_v108_revT_18Oct2016 (ONT). This preparative step included a DNA repair procedure to repair nicks, an end-repair step that also included dA-tailing of doublestranded DNA, ligation of sequencing adapters, AMPure XP bead purification, and tether protein attachment. Sequencing was performed as recommended by the manufacturer's guidelines using R9.4 flow cells (FLO-MIN106). MinION sequencing was controlled with MinKNOW software (v 18.01.6; ONT). Data was collected for approximately $26 \mathrm{~h}$. Base-calling was performed with Albacore (v 2.1.7; ONT). Only those reads meeting a minimum quality threshold, as determined by Albacore, were used for downstream analyses.

\section{Genome Assembly}

Canu v1.7 (c9ef921) was used with PacBio sequencing reads $>2 \mathrm{~kb}$ to assemble an initial 40-fold coverage. Canu was run with default parameters in grid mode (Sun Grid Engine) but with an estimated genome size of $6.8 \mathrm{Mb}$, a value based on other sequenced strains of $P$. aeruginosa. The completed assembly resulted in two closed (circular) contigs, one 6,822,869 bp, and another of 22,190 bp. Automated genome annotation with Prokka (Seemann, 2014) identified in the larger contig the replication initiation factor $\operatorname{dnaA}$, which promotes the unwinding of DNA at oriC. However, the smaller contig lacked an origin of replication, and contained only the ohb and hyb clusters along with uncharacterized genes and insertion sequence elements.

The characteristics of the small contig suggested the presence of a repeat, which may have been collapsed into a single copy during assembly. To test this hypothesis, BLAST-N (v. 2.6.0) was applied in homology searches of the Canu MHAP corrected reads using selected genes from the $o h b$ and $h y b$ clusters $(o h b B, h y b B)$, and the region adjoining the $h y b$ cluster $(m h q B)$. Although the $\mathrm{N} 50$ of filtered, preassembly, subread lengths was approximately $14 \mathrm{~kb}$, a small percentage of corrected reads extended beyond 24 $\mathrm{kb}$. Some of these longer reads contained $o h b B, h y b B$ and $m h q B$. Approximately $7.5 \mathrm{~kb}$ beyond the stop codon of $o h b B$, another region with near exact homology to $h y b B$ was observed, followed by the beginning sequence of $m h q B$ approximately $8.3 \mathrm{~kb}$ further. While this evidence was consistent with our hypothesis, none of the PacBio reads were sufficiently long to verify a complete repeat consisting of the three genes.

Guided by the premise that read length is an important determinant of genome assembly contiguity, we performed the same BLAST-N procedure using reads collected from the MinION sequencer. The N50 read length from the nanopore device was approximately $36.5 \mathrm{~kb}$ with many additional reads extending beyond $120 \mathrm{~kb}$. With this long-read information, we were able to confirm a repeat that contained $o h b B, h y b B$ and $m h q B$, and spanned approximately $36 \mathrm{~kb}$. To integrate this repeat into the large contig assembly, 40 of the highest quality MinION reads containing the complete repeat were aligned to the Canu assembly with Graphmap (Sovic et al., 2016) with the -C option enabled, to resolve coverage drops near the genome ends, should reads map near the ends. Racon (Vaser et al., 2017) was then used with uncorrected PacBio reads to generate a consensus and polish the entire assembly, including the repeated region.

The presence of the repeat and its position in the consensus assembly was confirmed with nucmer in the MUMmer-3.23 package (Kurtz et al., 2004). Lastly, paired-end, short-read Illumina data (approximately 51X coverage) were aligned to the consensus with bwa mem v0.7.12-r1039 (Li, 2013). Two rounds of polishing with Pilon v1.22 (Walker et al., 2014) were then applied to correct single base differences and small insertion and deletion events missed by consensus calling steps in Canu and Racon. This final polishing step resulted in 881 changes, reflecting a pre-correction agreement of 99.72 percent.

\section{Comparative Structural Analysis of Wild Type and Mutant ICEclc-JB2 by PCR}

A spontaneous mutant of $P$. aeruginosa JB2 deficient in growth on 2 -CBa was acquired by culturing on $1 \%$ glycerol as described previously (Hickey and Focht, 1990). Genomic DNA was extracted from the mutant by using an Illustra Bacterial Genomic DNA kit (GE Healthcare). PCR primers were designed to target regions in ICEclc-JB2 that were diagnostic of structure in the variable and core key regions (Table 1). All oligonucleotides used as primers were confirmed by BLAST against the $P$. aeruginosa JB2 genome as specific for the targeted location. PCR was done with ca. $50 \mathrm{ng}$ of gDNA template in OneTaq Hot Start Master Mix containing Standard Reaction Buffer (New England Biolabs) according to the manufacturer's recommendation. The thermal cycling program was an initial denaturation $\left(94^{\circ} \mathrm{C}, 30 \mathrm{~s}\right)$ followed by 30 cycles of: $94^{\circ} \mathrm{C}(15 \mathrm{~s}), 55^{\circ} \mathrm{C}$ or $62^{\circ} \mathrm{C}(15 \mathrm{~s})$ and $68^{\circ} \mathrm{C}$ (60 s). The final extension was $68^{\circ} \mathrm{C}$ for $5 \mathrm{~min}$. The annealing temperature used in Step 2 of the 30 cycle program was either $55^{\circ} \mathrm{C}$ or $62^{\circ} \mathrm{C}$ depending on the primer set (Table 1). All PCR assays were run in an Eppendorf Mastercycler Nexus Thermal Cycler. Analysis of PCR products was done by electrophoresis in agarose gels (1\% in TAE buffer) at $100 \mathrm{~V}$ for $3 \mathrm{~h}$. The size of PCR products was assessed by comparison to a GeneRuler $1 \mathrm{~kb}$ DNA ladder (ThermoFisher).

\section{Nucleotide Sequence Deposition}

The complete genome of $P$. aeruginosa JB2 is deposited in the National Center for Biotechnology Information Genbank database under accession number CP028917.1.

\section{RESULTS AND DISCUSSION}

\section{Overview of ICEclc-JB2 Structure and Comparisons to Other ICEclc}

In $P$. aeruginosa JB2, ICEclc (hereafter referred to as ICEclc-JB2) is located between bases $4,860,575$ to $4,983,845$ (123,270 bp) and is delimited by repeated tRNA Gly genes (76 bp). This structure 
TABLE 1 | PCR Primers used for deletion mapping ${ }^{a}$

\begin{tabular}{|c|c|c|}
\hline Set & Sequence $\left(5^{\prime}-3^{\prime}\right)$ & $\mathrm{T}_{\mathrm{m}}\left({ }^{\circ} \mathrm{C}\right)$ \\
\hline 1 & AGATTCTTGGGCGCTGTATC & 55 \\
\hline 1 & GGTTGATCAGTTCGTTGCAATAG & 55 \\
\hline 2 & CTCACCAGTGGCGTCAATAA & 55 \\
\hline 2 & GCATACGTCAGCAAGGATCA & 55 \\
\hline 3 & CGATCTCGGCGTCAAAGATT & 55 \\
\hline 3 & GGTAGCGCTTGTTCAGGTATAG & 55 \\
\hline 4 & TACCACCAGTGGGACTACAA & 55 \\
\hline 4 & CAGGTTCGGGAAGATCGAAAG & 55 \\
\hline 5 & CCACCGTTTGATGTTGGATTAG & 62 \\
\hline 5 & GAAGTAGTTCGACCCACCTATG & 62 \\
\hline 6 & CCTACACCGACAAAGACATCTAC & 55 \\
\hline 6 & TGGCTACCTTCAGCTTGTTC & 55 \\
\hline 7 & TGCGAAGGTCTCTCCTITCTA & 62 \\
\hline 7 & CCAAGCCCTCAGTTCGTTAAG & 62 \\
\hline 8 & CGATATCGCAGTCAGGAGAGA & 62 \\
\hline 8 & CTTCAGGCCATGGAAGACTATATG & 62 \\
\hline 9 & CAGCGCGATCAATGCAATAG & 62 \\
\hline 9 & GTCGCCAACATGCTCAATATC & 62 \\
\hline 10 & TCCTGAACAGCACCATCATC & 62 \\
\hline 10 & CAGTCATCGTCCACCCATTT & 62 \\
\hline 11 & GATTCTGCAAATCTGTCTCGGTA & 62 \\
\hline 11 & CTAGGGCAGTAAGTCGTTGATT & 62 \\
\hline
\end{tabular}

aSet number refers to primer binding site locations shown in Figure $3 A$.

of genomic integration is similar to that of ICEclc-LB400, which is also bounded by repeated tRNA Gly genes (79 bp). In contrast, only one end of ICEclc-B13 is bounded by a full tRNA Gly gene and the other end is delimited by a repeat of the last $18 \mathrm{bp}$ of the tRNA ${ }^{\text {Gly }}$ gene. The $P$. aeruginosa JB2 genome possess a single copy of ICEclc-JB2, in contrast to ICEclc-B13, which is present in two copies in the genome of P. knackmussii B13 (Miyazaki et al., 2015). Within the genome of strain JB2, all genes contained with ICEclc-JB2 were present only within that element.

ICEclc-JB2 exhibits the biomodal structure that is characteristic of ICEclc-B13 and ICEclc-LB400 with variable (cargo) genes encoding biodegradation functions segregated to one end, followed by $\mathrm{ca}$. $52 \mathrm{~kb}$ of core genes conserved within all three ICEclc (Figure 1). For ICEclc-B13 and ICEclc-LB400, the cluster of genes encoding aminophenol catabolism (amp) is a major component of the cargo area (29,300 bp), but is absent in ICEclc-JB2 (Figure 1). Two groups of cargo genes possessed by all three ICEclc are the cluster of genes annotated as encoding an anthranilate dioxygenase (Gaillard et al., 2006) immediately downstream of intB13 (Figure 1, Region 1), and the $c l c$ genes (Figure 1, Region 5). The latter of these are wellcharacterized as encoding enzymes of the catechol ortho-cleavage pathway $(c l c A B C D E)$ and a LysR element $(c l c R)$, which regulates expression of the $c l c$ operon (Ghosal and You, 1989). In contrast, the function of the putative anthranilate dioxygenase has yet to be determined, as none of the organisms possessing ICEclc have been reported to utilize anthranilate as a growth substrate.

The variable region of ICEclc-JB2 is distinct from that of other ICEclc in that its major block of biodegradation functions is present in a tandem repeat (Figures 1, 2). Each of the repeats contains the $o h b$ and $h y b$ genes (Figure 2), the former of these is shared with ICEclc-LB400 while the latter is not present in any other ICEclc yet reported. While the nucleotide sequence of the repeats is $99.9 \%$ identical, a number of indels occur in Repeat 1 some of which result in apparent frame-shifts and loss of several genes in Repeat 1 (Figure 2).

An element closely related to ICEclc, termed ICEXTD, has recently been characterized in several species and strains of Azoarcus (Zamarro et al., 2016). The close connection of ICEXTD to ICEclc is based on synteny of core functions (Zamarro et al., 2016). Also, like ICEclc, ICEXTD integrates at tRNA Gly genes, although it's attachment site spans the terminal 23-bp instead of $18 \mathrm{bp}$ as for ICEclc-B13 (Zamarro et al., 2016). Ecologically, ICEXTD plays a role similar to that of ICEclc, and endows upon the host the ability to utilize aromatic compounds as growth substrates (Zamarro et al., 2016). For ICEXTD, these compounds are toluene, $m$-xylene and cumene, the latter two of which support aerobic and anaerobic growth (Zamarro et al., 2016). While ICEXTD exhibits ecological and evolutionary similarities to ICEclc, ICEXTD differs significantly from ICEclc in structure in two respects. First, the core genes and cargo (termed adaption modules) are intermixed in ICEXTD, organization of core and cargo regions varies between ICEXTD that originate from different strains or species (Zamarro et al., 2016).

\section{The ohb Cluster of ICEclc-JB2}

Key growth substrates for $P$. aeruginosa JB2 are 2-CBa, 2,4-dichlorobenzoate, 2,5-dichlorobenzoate, and 2,3,5trichlorobenzoate, which are transformed by the $o h b A B$-encoded dioxygenase to catechol, 4-chlorocatechol (2,4- and 2,5dichlorobenzoate) and 3,5-dichlorocatechol, respectively (Hickey and Sabat, 2001). Prior work established that transformation of all ortho-chlorobenzoates and chlorocatechols is induced by growth on ortho-chlorobenzoates (Hickey and Focht, 1990). The clc cluster that occurs on ICEclc-JB2 encodes the enzymes adapted for funneling the chlorocatechols produced by the activity of OhbAB into pathways of central metabolism. Thus, physical linkage of $o h b$ and $c l c$ within ICEclc-JB2 makes the element a potentially efficient vehicle for conferring on a host the ability to utilize $o$-halobenzoates as carbon sources. Initial work with P. knackmussii B13 illustrated the importance of ICEclc in establishing the ability for utilization of 3-chlorobenzoate (Reineke and Knackmuss, 1980). But, in this case, the initial step of 3-chlorobenzoate transformation to 3-chlorocatechol was mediated by a dioxygenase that was not present within ICEclc-B13, but was instead encoded by an $x y l X Y Z$ ortholog located elsewhere in the P. knackmussii B13 genome (Miyazaki et al., 2015).

To date, only three records exist in Genbank for ohbAB orthologs in organisms other than $P$. aeruginosa JB2: Achromobacter xylosoxidans A8 (CP002288, pA8-1), Burkholderia xenovorans LB400 (CP000270) and Pseudomonas aeruginosa 142 (AF121870). With the exception of $P$. aeruginosa 142 , these records collectively establish that association with a self-transmissible MGE is a common feature of the ohb cluster 


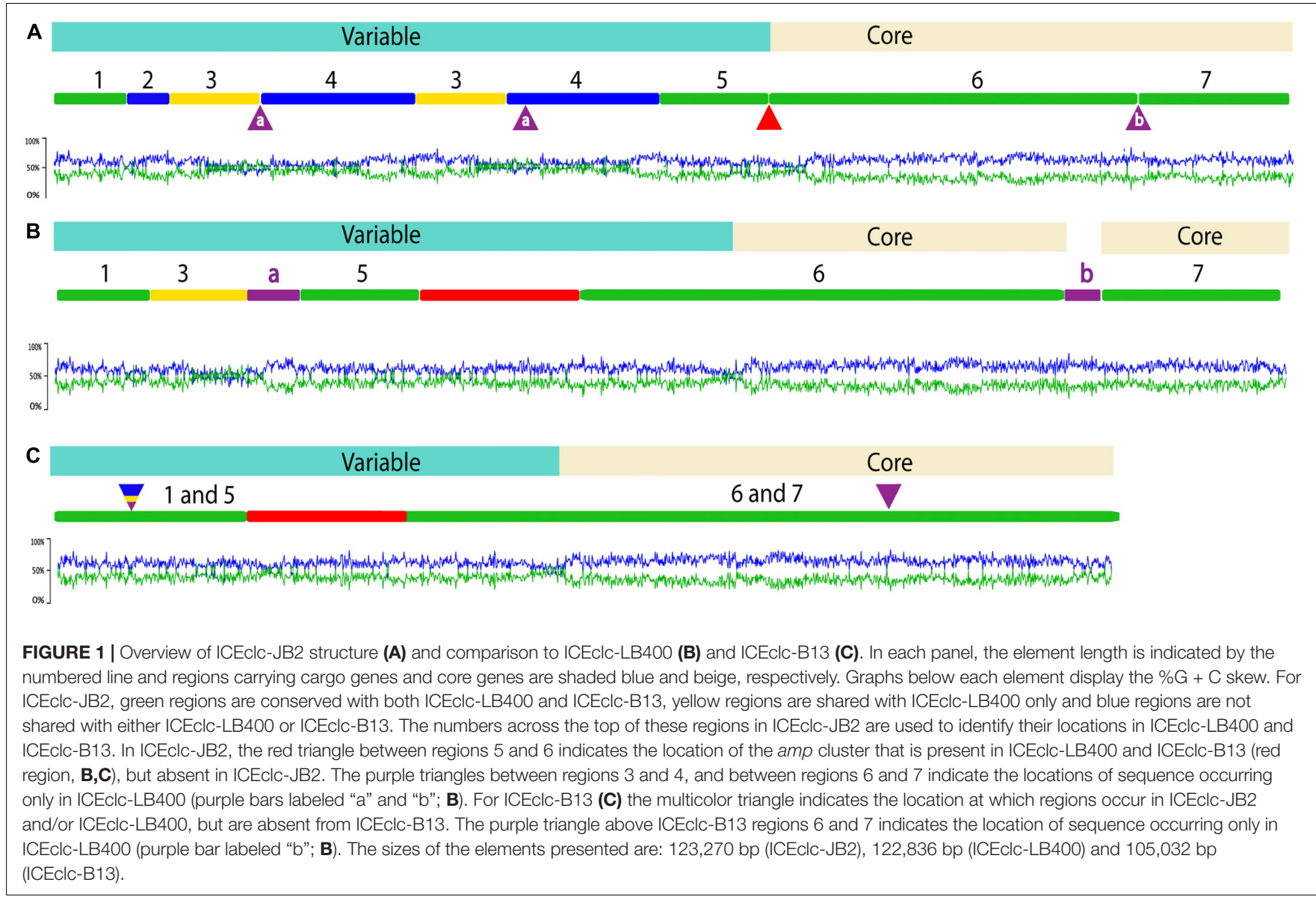

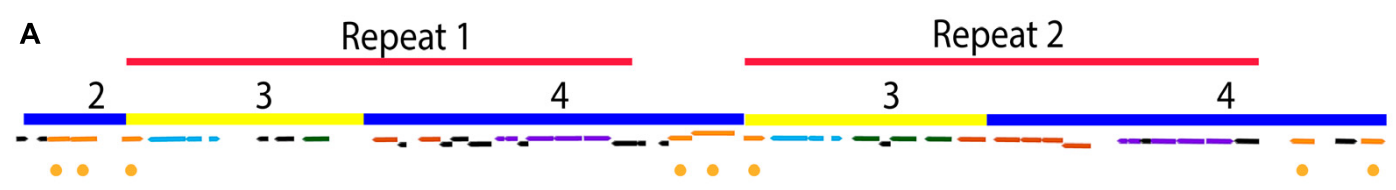

B $\quad$ Repeat 2
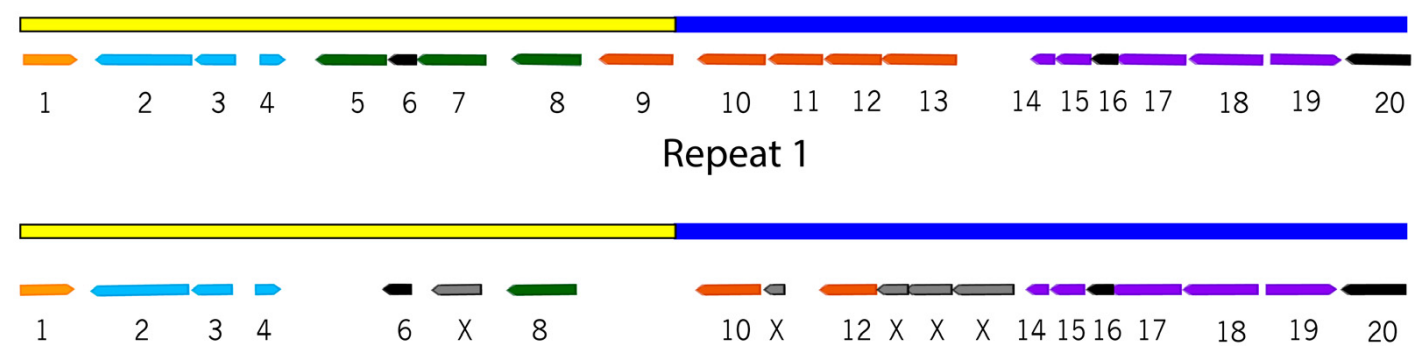

FIGURE 2 | Repeat regions in the ICEclc-JB2 cargo area. (A) Overview of the location and orientation of the repeat regions within ICEclc-JB2 cargo area. The color-coding and numbering of bars is as described in Figure 1. The locations of transposases/insertion sequence elements are indicated by gold circles. (B) Detail of the repeat regions showing gene structure, numbering across the top of each repeat is the base pair location in the $P$. aeruginosa JB2 genome. Genes are numbered 1-20 based on structure in Repeat 2. Color-coding of genes is as follows: predicted transposase (gold; gene 1), ohbBAR (light blue; genes 2-4, respectively), predicted catabolic enzymes (green; genes 5, 7, 8), hybIHGEF (orange, genes 9-13, respectively), hybDCABR (purple; genes 14, 15, 17-19 respectively), hypothetical (black; genes $6,16,20$ ). In Repeat 1, frame shifts result in the absence of genes 5 and 9 and the replacement of genes 7,11 , and 13 with fragmented ORFs (indicated by “'”). In (B), Repeat 2 spans (left to right) genome positions 4,949,315-4,933,315 and Repeat 1 spans genome positions $4,971,315-4,955,315$ (left to right). 
although the nature of the element is variable: ICEclc in the case of $P$. aeruginosa JB2 and B. xenovorans LB400 and a $98 \mathrm{~kb}$ IncP1- $\beta$ plasmid in the case of $A$. xylosoxidans A8. For $P$. aeruginosa 142, an unequivocal assessment of association with an MGE is not possible as the current data for strain 142 is limited to a partial record of 6,052 bp that encompasses only the ohb genes and flanking regions. However, based on what is known about ohb in the other organisms discussed here, association of $o h b$ with ICEclc or other MGE might be expected for $P$. aeruginosa 142 .

The ohb clusters are similar in that $o h b A$ is immediately adjoined downstream by a divergently oriented coding sequence $(o h b R)$ for which an IclR-type transcriptional regulatory element is predicted. For ICEclc-JB2, ICEclc-LB400 and pA81, ohbR is 255 bp with a predicted polypeptide of 85 amino acids. In contrast, for $P$. aeruginosa 142 , ohbR is $717 \mathrm{bp}$ with a predicted polypeptide of 239 amino acids. The predicted polypeptides of both the long and short versions of $o h b R$ possess the N-terminal LTTR motif characteristic of IclR elements, which is required for recognition of DNA binding sites (Tropel and van der Meer, 2004). However, IclR function also requires C-terminal structure essential for the binding of effector molecules and for the multimerization of IclR polypeptides to tetramers, the supramolecular structure active in binding DNA (Tropel and van der Meer, 2004). Thus, while regulation of $o h b A B$ has not been empirically determined for any organism, it appears that the predicted OhbR of $P$. aeruginosa 142 could play a role in this process, as it possesses the $\mathrm{N}$-terminal and C-terminal structure requisite for function as an IclR regulator. But, the apparently truncated version of OhbR predicted in the other three cases would not be functional according to criteria established for IclR elements (Tropel and van der Meer, 2004). While the genome of strain JB2 has only one copy of $o h b R$, it possesses numerous other predicted IclR elements that could affect $o h b A B$ expression; empirical research is needed to delineate the regulatory system for $o h b A B$.

Of the four organisms that possess ohbAB, B. xenovorans LB400 is the only one for which growth on $o$-chlorobenzoates (or any chlorobenzoic acid) has not yet been demonstrated. The novel phenotype of B. xenovorans LB400 [a.k.a. Pseudomonas sp. LB400 (Bopp, 1986), Burkholderia sp. LB400 (Bartels et al., 1999), Burkholderia fungorum LB400 (Marx et al., 2004), Paraburkholderia xenovorans LB400, (Sawana et al., 2014)] is a relatively broad spectrum of PCB congeners that it transforms by cometabolism (i.e., growth on biphenyl) to a variety of products. Cometabolism by B. xenovorans LB400 of some lower chlorinated PCBs yields chlorobenzoic acids, but strain LB400 does not grow on chlorobenzoic acids, which limits growth on PCBs (Potrawfke et al., 1998). In particular, specific testing with 2-CBa as a sole carbon source failed to elicit growth by B. xenovorans LB400 (Rodrigues et al., 2006). It should be noted that these studies pre-dated genome sequencing of $B$. xenovorans LB400 (Chain et al., 2006), and the existence of ICEclc-LB400 (and the ohb genes) in B. xenovorans LB400 was unknown. Thus, in an effort to improve the PCB-degradation capabilities of B. xenovorans LB400 by transforming it to a strain that could grow $o$-chlorobenzoates (and thus grow on some PCBs),
ohbRAB were cloned from $P$. aeruginosa 142 (Tsoi et al., 1999) and introduced to $B$. xenovorans LB400, yielding a transformant designated as B. xenovorans $\mathrm{LB} 400(\mathrm{ohb})$ that was capable of growth with 2-CBa (Rodrigues et al., 2006).

It's unclear why introduction of $o h b R A B$ to $B$. xenovorans LB400, an organism that natively possessed the ohb genes, was necessary to enable its growth on 2-CBa. Possibly, the full-length ohbR introduced with the $P$. aeruginosa 142 ohb cluster complemented the truncated $o h b R$ native to ICEclc-B13, and thereby provided a regulatory function that alleviated a restriction on gene expression. But, if so, that would mean that two different organisms ( $P$. aeruginosa JB2 and A. xylosoxidans A8) that also possess a truncated $o h b R$, yet grow on 2$\mathrm{CBa}$, can complement the function of the truncated $o h b R$, whereas B. xenovorans LB400 cannot. While its unknown why $P$. aeruginosa JB2, A. xylosoxidans A8 and B. xenovorans LB400 differ in the utilization of $2-\mathrm{CBa}$, the divergent phenotypes of these three organisms underscore the importance of the host background as a modulator of the expression of functions encoded by ICEclc specifically, and MGE in general.

The physical linkage of genes encoding $o$-chlorobenzobenzoate $(o h b A B)$ and $o$-hydroxybenzoate (hybABCD) metabolism was implied in previous studies, wherein these phenotypes were acquired simultaneously by bacteria mated with P. aeruginosa JB2 (Hickey et al., 2001). The present description of ICEclc-JB2 provides insight into the associations with MGE that may have been involved in those gene transfers. The hyb region (Figure 2) includes a LysR regulatory element (hybR), a ring-hydroxylating monooxygenase (hybABCD, transforms salicylate to gentisate), and the components of an ABC-type uptake $(h y b E F G H I)$. The $h y b$ cluster is distinct in exhibiting a $\% \mathrm{G}+\mathrm{C}$ content that is significantly lower than that of the neighboring $o h b$ genes indicating an origin divergent from that of ohb (Figure 1).

\section{ICEclc-JB2 Lacks Genes Encoding Key Regulatory Functions}

Functions have been identified within the core region of ICEclcB13, which control its stabilization and mobilization (Minoia et al., 2008; Miyazaki and van der Meer, 2011; Pradervand et al., 2014; Delavat et al., 2016). A key feature is an operon of three genes that effects global regulation of transfer initiation (Pradervand et al., 2014). These genes are designated as $t c i R$, $\operatorname{marR}, m f s R$, and encode a LysR-type activator (TciR), a MarRtype regulator (MarR) and a TetR-type repressor (MfsR), respectively. The $t c i R-m a r R-m f s R$ operon also exists in ICEclcLB400 and is located at same position as in ICEclc-B13, the junction between the $c l c$ region (Region 5, Figure 1) and the amp cluster (red segment, Figure 1). However, this location is contained with a $c a .28 \mathrm{~kb}$ region of ICEclc-B13/LB400 that is absent in ICEclc-JB2, which corresponds to the region spanning ORF18502 to ORF4677 in ICEclc-B13 (Gaillard et al., 2006). The tciR-marR-mfsR genes do not exist elsewhere in the strain JB2 genome. The absence of $t c i R-m a r R-m f s R$ from ICEclc-JB2 indicates that either other genes are the functional equivalent of this operon and/or the regulation of ICEclc-JB2 
transfer occurs by mechanisms different from those established for ICEclc-B13. Identification of additional ICEclc relatives is needed to determine the frequency with which the tciR-marR$m f s R$ cluster is present or absent from these elements. For ICEclcJB2, and other ICEclc relatives lacking $t c i R-m a r R-m f s R$, studies are needed to elucidate the systems that control stabilization and mobilization of the elements.

\section{Independent Mobilization of ICEclc-JB2 Cargo Genes}

Earlier studies with $P$. aeruginosa JB2 demonstrated that the spontaneous loss of the 2-CBa utilization phenotype occurred when strain was cultured on a substrate such as glycerol (Hickey and Focht, 1990). The data presented in this report supports two hypotheses about the process(es) that gave rise to the mutant phenotype. Given that ICEclc controls its own excision, one hypothesis is that the entire element is lost from the genome. Alternatively, the repeats harboring the ohb genes are neighbored by multiple transposases/insertion sequence elements (Figure 2A) and its possible ohb loss results from excision of these elements. In this case, elimination of $o h b$ would occur independently of ICEclc-JB2 as a whole, which would remain in the genome absent the ohb genes.

To test these two hypotheses, a 2-CBa-deficient mutant (derived from a glycerol-grown culture) was examined by PCR to determine the presence/absence of regions located in ICEclc core regions, genes within the repeats and transposable elements that border the repeats (Figure 3). The wild type and mutant both gave expected PCR products for regions targeting either end of ICEclc-JB2 (Figure 3, primer sets 1, 11) and to primers targeting clc genes in the conserved region adjacent to the repeat region (Figure 3, primer sets 8-10). The mutant was also positive in PCR for primer pair 2, which targeted an insertion sequence element that was present at either end of the repeat region (Figure 3 ). In contrast, primers targeting $o h b B, h y b B$ and a gene between $o h b B$ and $h y b B$ (gene 8 , Figure 2B) were all negative in the mutant (Figure 3, primer sets 4-6). Thus, the boundaries of the excised region lay between the insertion sequence element targeted by primer pair 2 (bases 4,930,538-4,974,802).

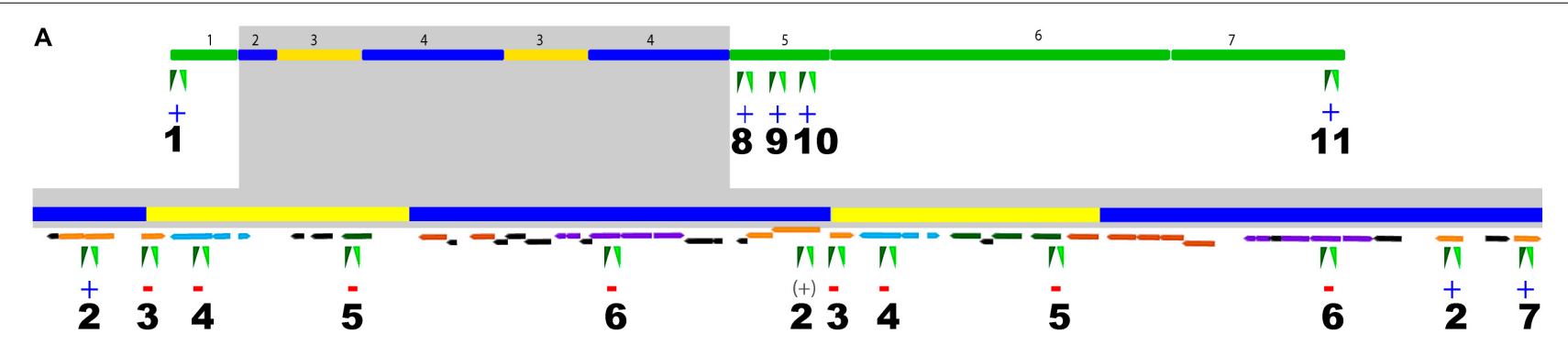

B

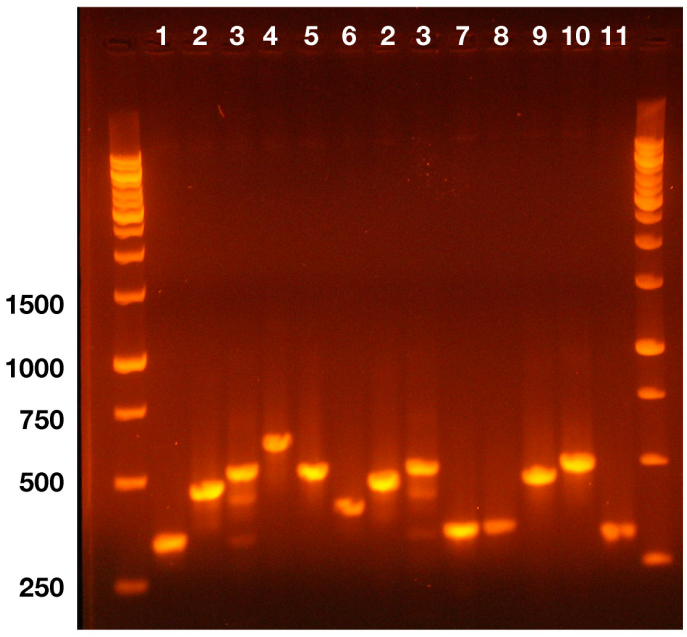

C

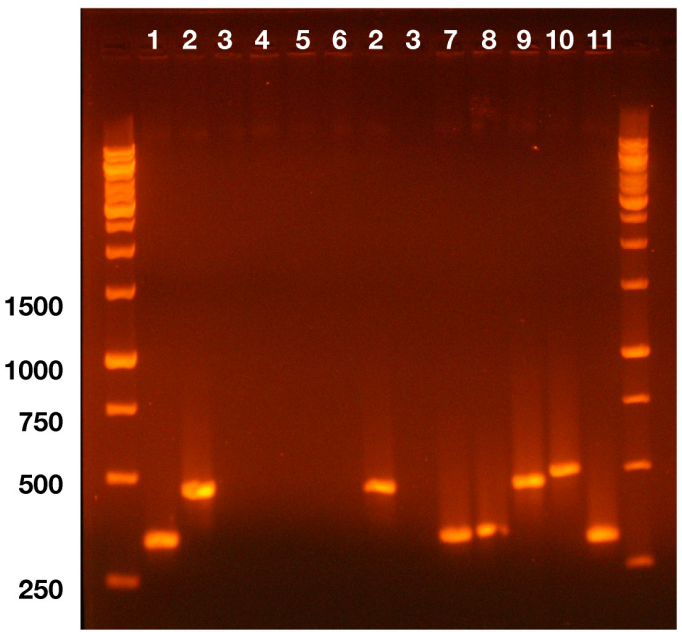

FIGURE 3 | PCR Analysis of ICEclc-JB2 structure in wild type P. aeruginosa JB2 and a spontaneous mutant of strain JB2 deficient in growth on 2-CBa. (A) Map of ICEclc-JB2 displaying locations of 11 PCR primer pairs used to probe its structure. The gray box indicates expanded detail of the repeat region. Color-coding of genes follows is as used in Figure 2. The symbols "+" or "-" beneath numbers 1-11 indicate the presence or absence, respectively of a PCR product in the mutant for the indicated primer pair. For primer pair 2, the symbol "( + ") is placed beneath its location between repeats 1 and 2 to indicate that the product observed in the mutant was likely derived from one or both of its other two binding sites that are located at the termini of the repeats. (B) Agarose gel of PCR products from the wild type. (C) Agarose gel of PCR products from the mutant. Numbering across the top of each gel corresponds to the primer pair location indicated in (A). Numbering along the left side of gels indicates the size of the five bottom bands in the DNA ladder. 
Collectively, results of the PCR analysis support the hypothesis that the mutant phenotype resulted from loss of the ohb genes from ICEclc-JB2, not loss of ICEclc-JB2 as a whole. Loss of the $o h b$ genes was associated with excision of the entire repeat region that encompassed a total of $c a .44 \mathrm{~kb}$. Thus, more than half of the cargo region can excise independently of ICEclc-JB2, and possibly involves the transposases/insertion sequence elements that are associated with the repeat region. Loss of the repeat results in the loss of key, selective phenotypes encoded by ICEclc. In the short term, loss of the repeat region potentially makes it less useful to a host. However, the reduced version of ICEclcJB2 would now have much unused physical capacity for cargo, which it could use to gain new genes (phenotypes) that might be beneficial to a future host and, consequently, to the long-term survival of ICEclc.

\section{CONCLUSION}

The present report establishes a new ICE, ICEclc-JB2, which is closely related to ICEclc-B13 and ICEclc-LB400. All three elements are ecologically similar in that they potentially confer on the host biodegradation phenotypes. For ICEclc-JB2 unique

\section{REFERENCES}

Banuelos-Vazquez, L. A., Tejerizo, G. T., and Brom, S. (2017). Regulation of conjugative transfer of plasmids and integrative conjugative elements. Plasmid 91, 82-89. doi: 10.1016/j.plasmid.2017.04.002

Bartels, F., Backhaus, S., Moore, E. R. B., Timmis, K. N., and Hofer, B. (1999). Occurrence and expression of glutathione-S-transferase-encoding $b p h K$ genes in Burkholderia sp strain LB400 and other biphenyl-utilizing bacteria. Microbiology 145, 2821-2834. doi: 10.1099/00221287-145-102821

Bie, L. Y., Wu, H., Wang, X. H., Wang, M. Y., and Xu, H. (2017). Identification and characterization of new members of the SXT/R391 family of integrative and conjugative elements (ICEs) in Proteus mirabilis. Int. J. Antimicrob. Agents 50, 242-246. doi: 10.1016/j.ijantimicag.2017.01.045

Bopp, L. H. (1986). Degradation of highly chlorinated PCBs by Pseudomonas strain LB400. J. Indust. Microbiol. 1, 23-29. doi: 10.1007/BF0156 9413

Chain, P. S. G., Denef, V. J., Konstantinidis, K. T., Vergez, L. M., Agullo, L., Reyes, V. L., et al. (2006). Burkholderia xenovorans LB400 harbors a multireplicon, 9.73-Mbp genome shaped for versatility. Proc. Natl. Acad. Sci. U.S.A. 103, 15280-15287. doi: 10.1073/pnas.0606924103

Chong, C. S., Sabir, D. K., Lorenz, A., Bontemps, C., Andeer, P., Stahl, D. A., et al. (2014). Analysis of the xplAB-containing gene cluster involved in the bacterial degradation of the explosive Hexahydro-1,3,5-Trinitro-1,3,5Triazine. Appl. Environ. Microbiol. 80, 6601-6610. doi: 10.1128/AEM.018 $18-14$

Chowdhury, P. R., Scott, M., Worden, P., Huntington, P., Hudson, B., Karagiannis, T., et al. (2016). Genomic islands 1 and 2 play key roles in the evolution of extensively drug-resistant ST235 isolates of Pseudomonas aeruginosa. Open Biol. 6:150175. doi: 10.1098/rsob.150175

Chuzeville, S., Auger, J. P., Dumesnil, A., Roy, D., Lacouture, S., Fittipaldi, N., et al. (2017). Serotype-specific role of antigen I/II in the initial steps of the pathogenesis of the infection caused by Streptococcus suis. Vet. Res. 48:39. doi: 10.1186/s13567-017-0443-4

Clawson, M. L., Murray, R. W., Sweeney, M. T., Apley, M. D., Dedonder, K. D., Capik, S. F., et al. (2016). Genomic signatures of Mannheimia haemolytica that associate with the lungs of cattle with respiratory disease, an integrative conjugative element, and antibiotic resistance genes. BMC Genomics 17:982. doi: $10.1186 / \mathrm{s} 12864-016-3316-8$ the characteristics endowed are the utilization of $o$-halobenzoates and $o$-hydroxybenzoate as growth substrates. While ICEclcJB2 is highly similar to ICEclc-B13 and ICEclc-LB400, it lacks key a regulatory gene that is present in the other two ICEclc, which controls excision from the host. Thus, the mechanisms regulating intracellular behavior of ICEclc-JB2 may differ from that of its close relatives. More than half of the cargo genes carried by ICEclc-JB2 can excise independently from the element, potentially providing evolutionary flexibility for the element.

\section{AUTHOR CONTRIBUTIONS}

CO, SV, VG, MB, JW, DP, JH, and WH all contributed to experimental work, data analysis, and preparation of the manuscript.

\section{FUNDING}

This project was supported by funding from the O.N. Allen Professorship in Soil Microbiology and by USDA Hatch Project WIS01929.

Colombi, E., Straub, C., Kunzel, S., Templeton, M. D., Mccann, H. C., and Rainey, P. B. (2017). Evolution of copper resistance in the kiwifruit pathogen Pseudomonas syringae pv. actinidiae through acquisition of integrative conjugative elements and plasmids. Environ. Microbiol. 19, 819-832. doi: 10. $1111 / 1462-2920.13662$

Cury, J., Touchon, M., and Rocha, E. P. C. (2017). Integrative and conjugative elements and their hosts: composition, distribution and organization. Nucleic Acids Res. 45, 8943-8956. doi: 10.1093/nar/gkx607

Delavat, F., Mitri, S., Pelet, S., and Van Der Meer, J. R. (2016). Highly variable individual donor cell fates characterize robust horizontal gene transfer of an integrative and conjugative element. Proc. Natl. Acad. Sci. U.S.A. 113, E3375E3383. doi: 10.1073/pnas.1604479113

Delavat, F., Miyazaki, R., Carraro, N., Pradervand, N., and Van Der Meer, J. R. (2017). The hidden life of integrative and conjugative elements. FEMS Microbiol. Rev. 41, 512-537. doi: 10.1093/femsre/fux008

Diaz, E. (2004). Bacterial degradation of aromatic pollutants: a paradigm of metabolic versatility. Int. Microbiol. 7, 173-180.

Field, J. A., and Sierra-Alvarez, R. (2008). Microbial transformation and degradation of polychlorinated biphenyls. Environ. Pollut. 155, 1-12. doi: 10. 1016/j.envpol.2007.10.016

Gaillard, M., Vallaeys, T., Vorholter, F. J., Minoia, M., Werlen, C., Sentchilo, V., et al. (2006). The $c l c$ element of Pseudomonas sp strain B13, a genomic island with various catabolic properties. J. Bacteriol. 188, 1999-2013. doi: 10.1128/JB. 188.5.1999-2013.2006

Ghosal, D., and You, I. S. (1989). Operon structure and nucleotide homology of the chlorocatechol oxidation genes of plasmids pJP4 and pAC27. Gene 83, 225-232. doi: 10.1016/0378-1119(89)90108-X

Harmer, C. J., Hamidian, M., and Hall, R. M. (2017). pIP40a, a type 1 IncC plasmid from 1969 carries the integrative element GIsul2 and a novel class II mercury resistance transposon. Plasmid 92, 17-25. doi: 10.1016/j.plasmid.2017. 05.004

Hickey, W. J., Chen, S. C., and Zhao, J. C. (2012). The phn island: a new genomic island encoding catabolism of polynuclear aromatic hydrocarbons. Front. Microbiol. 3:125. doi: 10.3389/fmicb.2012.00125

Hickey, W. J., and Focht, D. D. (1990). Degradation of monohalogenated, dihalogenated and trihalogenated benzoic-acids by Pseudomonas aeruginosa JB2. Appl. Environ. Microbiol. 56, 3842-3850.

Hickey, W. J., and Sabat, G. (2001). Integration of matrix-assisted laser desorption ionization-time of flight mass spectrometry and molecular 
cloning for the identification and functional characterization of mobile ortho-halobenzoate oxygenase genes in Pseudomonas aeruginosa strain JB2. Appl. Environ. Microbiol. 67, 5648-5655. doi: 10.1128/AEM.67.12.5648-5655. 2001

Hickey, W. J., Sabat, G., Yuroff, A. S., Arment, A. R., and Perez-Lesher, J. (2001). Cloning, nucleotide sequencing, and functional analysis of a novel, mobile cluster of biodegradation genes from Pseudomonas aeruginosa strain JB2. Appl. Environ. Microbiol. 67, 4603-4609. doi: 10.1128/AEM.67.10.4603-4609. 2001

Hickey, W. J., Searles, D. B., and Focht, D. D. (1993). Enhanced mineralization of polychlorinated-biphenyls in soil inoculated with chlorobenzoate-degrading bacteria. Appl. Environ. Microbiol. 59, 1194-1200.

Johnson, C. M., and Grossman, A. D. (2015). Integrative and conjugative elements (ICEs): what they do and how they work. Annu. Rev. Genet. 49, 577-601. doi: 10.1146/annurev-genet-112414-055018

Kurtz, S., Phillippy, A., Delcher, A. L., Smoot, M., Shumway, M., Antonescu, C., et al. (2004). Versatile and open software for comparing large genomes. Genome Biol. 5:R12. doi: 10.1186/gb-2004-5-2-r12

Leon-Sampedro, R., Novais, C., Peixe, L., Baquero, F., and Coque, T. M. (2016). Diversity and evolution of the Tn5801-tet(M)-like integrative and conjugative elements among Enterococcus, Streptococcus, and Staphylococcus. Antimicrob. Agents Chemother. 60, 1736-1746. doi: 10.1128/AAC.018 64-15

Li, H. (2013). Aligning sequence reads, clone sequences and assembly contigs with BWA-MEM. arXiv:1303.3997 [Preprint].

Ling, J., Wang, H., Wu, P., Li, T., Tang, Y., Naseer, N., et al. (2016). Plant nodulation inducers enhance horizontal gene transfer of Azorhizobium caulinodans symbiosis island. Proc. Natl. Acad. Sci. U.S.A. 113, 13875-13880. doi: 10.1073/ pnas. 1615121113

Marx, C. J., Miller, J. A., Chistoserdova, L., and Lidstrom, M. E. (2004). Multiple formaldehyde oxidation/detoxification pathways in Burkholderia fungorum LB400. J. Bacteriol. 186, 2173-2178. doi: 10.1128/JB.186.7.2173-2178. 2004

Minoia, M., Gaillard, M., Reinhard, F., Stojanov, M., Sentchilo, V., and Van Der Meer, J. R. (2008). Stochasticity and bistability in horizontal transfer control of a genomic island in Pseudomonas. Proc. Natl. Acad. Sci. U.S.A. 105, 20792-20797. doi: 10.1073/pnas.0806164106

Miyazaki, R., Bertelli, C., Benaglio, P., Canton, J., De Coi, N., Gharib, W. H., et al. (2015). Comparative genome analysis of Pseudomonas knackmussii $\mathrm{B} 13$, the first bacterium known to degrade chloroaromatic compounds. Environ. Microbiol. 17, 91-104. doi: 10.1111/1462-2920. 12498

Miyazaki, R., and van der Meer, J. R. (2011). A dual functional origin of transfer in the ICEclc genomic island of Pseudomonas knackmussii B13. Mol. Microbiol. 79, 743-758. doi: 10.1111/j.1365-2958.2010.07484.x

Olaitan, A. O., Diene, S. M., Assous, M. V., and Rolain, J. M. (2016). Genomic plasticity of multidrug-resistant NDM-1 positive clinical isolate of Providencia rettgeri. Genome Biol. Evol. 8, 723-728. doi: 10.1093/gbe/ evv195

Pathak, A., Chauhan, A., Blom, J., Indest, K. J., Jung, C. M., Stothard, P., et al. (2016). Comparative genomics and metabolic analysis reveals peculiar characteristics of Rhodococcus opacus strain M213 particularly for naphthalene degradation. PLoS One 11:e0161032. doi: 10.1371/journal.pone.016 1032

Perezlesher, J., and Hickey, W. J. (1995). Use of an s-triazine nitrogensource to select for and isolate a recombinant chlorobenzoate-degrading Pseudomonas. FEMS Microbiol. Lett. 133, 47-52. doi: 10.1016/0378-1097(95)00 $333-\mathrm{Z}$

Potrawfke, T., Lohnert, T. H., Timmis, K. N., and Wittich, R. M. (1998). Mineralization of low-chlorinated biphenyls by Burkholderia sp. strain LB400 and by a two membered consortium upon directed interspecies transfer of chlorocatechol pathway genes. Appl. Microbiol. Biotechnol. 50, 440-446. doi: $10.1007 / \mathrm{s} 002530051318$

Pradervand, N., Sulser, S., Delavat, F., Miyazaki, R., Lamas, I., and Van Der Meer, J. R. (2014). An operon of three transcriptional regulators controls horizontal gene transfer of the Integrative and Conjugative Element ICE $c / c$ in Pseudomonas knackmussii B13. PLoS Genet. 10:e1005130. doi: 10.1371/journal. pgen. 1005130
Reineke, W., and Knackmuss, H. J. (1980). Hybrid pathway for chlorobenzoate metabolism in Pseudomonas sp-B13 derivatives. J. Bacteriol. 142, 467-473.

Rodrigues, J. L. M., Kachel, C. A., Aiello, M. R., Quensen, J. F., Maltseva, O. V., Tsoi, T. V., et al. (2006). Degradation of Aroclor 1242 dechlorination products in sediments by Burkholderia xenovorans LB400(ohb) and Rhodococcus sp strain RHA1(fcb). Appl. Environ. Microbiol. 72, 2476-2482. doi: 10.1128/AEM.72.4. 2476-2482.2006

Ryan, M. P., Armshaw, P., O’halloran, J. A., and Pembroke, J. T. (2017). Analysis and comparative genomics of R997, the first SXT/R391 integrative and conjugative element (ICE) of the Indian Sub-Continent. Sci. Rep. 7:8562. doi: 10.1038/s41598-017-08735-y

Sawana, A., Adeolu, M., and Gupta, R. S. (2014). Molecular signatures and phylogenomic analysis of the genus Burkholderia: proposal for division of this genus into the emended genus Burkholderia containing pathogenic organisms and a new genus Paraburkholderia gen. nov harboring environmental species. Front. Genet. 5:429. doi: 10.3389/fgene.2014.00429

Seemann, T. (2014). Prokka: rapid prokaryotic genome annotation. Bioinformatics 30, 2068-2069. doi: 10.1093/bioinformatics/btu153

Shintani, M., Takahashi, Y., Yamane, H., and Nojiri, H. (2010). The behavior and significance of degradative plasmids belonging to Inc groups in Pseudomonas within natural environments and microcosms. Microbes Environ. 25, 253-265. doi: 10.1264/jsme2.ME10155

Sovic, I., Sikic, M., Wilm, A., Fenlon, S. N., Chen, S., and Nagarajan, N. (2016). Fast and sensitive mapping of nanopore sequencing reads with GraphMap. Nat. Commun. 7:11307. doi: 10.1038/ncomms11307

Suenaga, H., Fujihara, H., Kimura, N., Hirose, J., Watanabe, T., Futagami, T., et al. (2017). Insights into the genomic plasticity of Pseudomonas putida KF715, a strain with unique biphenyl-utilizing activity and genome instability properties. Environ. Microbiol. Rep. 9, 589-598. doi: 10.1111/1758-2229. 12561

Sugimoto, Y., Suzuki, S., Nonaka, L., Boonla, C., Sukpanyatham, N., Chou, H. Y., et al. (2017). The novel $m e f(\mathrm{C})-m p h(\mathrm{G})$ macrolide resistance genes are conveyed in the environment on various vectors. J. Glob. Antimicrob. Resist. 10, 47-53. doi: 10.1016/j.jgar.2017.03.015

Top, E. M., and Springael, D. (2003). The role of mobile genetic elements in bacterial adaptation to xenobiotic organic compounds. Curr. Opin. Biotechnol. 14, 262-269. doi: 10.1016/S0958-1669(03)00066-1

Top, E. M., Springael, D., and Boon, N. (2002). Catabolic mobile genetic elements and their potential use in bioaugmentation of polluted soils and waters. FEMS Microbiol. Ecol. 42, 199-208. doi: 10.1111/j.1574-6941.2002.tb01 009.x

Tropel, D., and van der Meer, J. R. (2004). Bacterial transcriptional regulators for degradation pathways of aromatic compounds. Microbiol. Mol. Biol. Rev. 68, 474-500. doi: 10.1128/MMBR.68.3.474-500.2004

Tsoi, T. V., Plotnikova, E. G., Cole, J. R., Guerin, W. F., Bagdasarian, M., and Tiedje, J. M. (1999). Cloning, expression, and nucleotide sequence of the Pseudomonas aeruginosa 142 ohb genes coding for oxygenolytic ortho dehalogenation of halobenzoates. Appl. Environ. Microbiol. 65, 2151-2162.

van der Meer, J. R., and Sentchilo, V. (2003). Genomic islands and the evolution of catabolic pathways in bacteria. Curr. Opin. Biotechnol. 14, 248-254. doi: $10.1016 /$ S0958-1669(03)00058-2

Vanneste, J. L. (2017). The scientific, economic, and social impacts of the New Zealand outbreak of bacterial canker of Kiwifruit (Pseudomonas syringae pv. actinidiae). Ann. Rev. Phytopathol. 55, 377-399. doi: 10.1146/annurevphyto-080516-035530

Vaser, R., Sovic, I., Nagarajan, N., and Sikic, M. (2017). Fast and accurate de novo genome assembly from long uncorrected reads. Genome Res. 27, 737-746. doi: $10.1101 /$ gr.214270.116

Walker, B. J., Abeel, T., Shea, T., Priest, M., Abouelliel, A., Sakthikumar, S., et al. (2014). Pilon: an integrated tool for comprehensive microbial variant detection and genome assembly improvement. PLoS One 9:e112963. doi: 10.1371/journal. pone. 0112963

Wang, P. X., Zeng, Z. S., Wang, W. Q., Wen, Z. L., Li, J., and Wang, X. X. (2017). Dissemination and loss of a biofilm-related genomic island in marine Pseudoalteromonas mediated by integrative and conjugative elements. Environ. Microbiol. 19, 4620-4637. doi: 10.1111/1462-2920. 13925 
Wozniak, R. A., and Waldor, M. K. (2010). Integrative and conjugative elements: mosaic mobile genetic elements enabling dynamic lateral gene flow. Nat. Rev. Microbiol. 8, 552-563. doi: 10.1038/nrmicro2382

Zamarro, M. T., Martin-Moldes, Z., and Diaz, E. (2016). The ICEXTD of Azoarcus sp CIB, an integrative and conjugative element with aerobic and anaerobic catabolic properties. Environ. Microbiol. 18, 5018-5031. doi: 10.1111/14622920.13465

Zhou, K. X., Xie, L. Y., Han, L. Z., Guo, X. K., Wang, Y., and Sun, J. Y. (2017). ICESag37, a novel integrative and conjugative element carrying antimicrobial resistance genes and potential virulence factors in Streptococcus agalactiae. Front. Microbiol. 8:1921. doi: 10.3389/fmicb.2017.01921
Conflict of Interest Statement: The authors declare that the research was conducted in the absence of any commercial or financial relationships that could be construed as a potential conflict of interest.

Copyright (c) 2018 Obi, Vayla, de Gannes, Berres, Walker, Pavelec, Hyman and Hickey. This is an open-access article distributed under the terms of the Creative Commons Attribution License (CC BY). The use, distribution or reproduction in other forums is permitted, provided the original author(s) and the copyright owner(s) are credited and that the original publication in this journal is cited, in accordance with accepted academic practice. No use, distribution or reproduction is permitted which does not comply with these terms. 